\title{
SPECTROSCOPY AND POLARIMETRY OF THE R AQUARII SYSTEM
}

\author{
Hugo E. Schwarz \\ Mullard Space Science Laboratory, Holmbury St. Mary, Dorking, Surrey, RH5 6NT, UK \\ Colin Aspin \\ Royal Observatory, Blackford Hill, Edinburgh EH9 3HJ, UK
}

\begin{abstract}
Optical spectroscopy is used to derive the electron temperature and density of the jet of R Aqr. We present polarimetric data which shows that the degree of polarization in $R$ Aqr can vary by up to two orders of magnitude and that this variation is correlated with the phase of the Mira. The polarization angle also varies with the phase of observation.
\end{abstract}

\section{INTRODUCTION}

$\mathrm{R}$ Aqr is a peculiar symbiotic with a 387 day Mira as cool component. The system has a binary period of 44 years and (perhaps) nova-like mass ejections at irregular intervals. The binary is embedded in a complex nebula consisting of three inner knots forming a curved jet out to $\sim 7$ arcseconds and a much larger nebula extending over $\sim 2$ arcminutes in the shape of two intersecting arcs.

Observations of R Aqr have been made at radio (Kafatos and Michalitsianos, 1982), infra-red (Stein et al., 1969), optical (Mauron et al., 1985; Wallerstein, 1986), ultra-violet (Kafatos et al., 1986) and X-ray wavelengths (Baratta et al., 1985). Broad-band polarimetric observations have been made by several authors (e.g. Serkowski, 1971) and recently a high resolution polarization spectrum has been obtained by Aspin et al. (1985).

\section{SPECTROSCOPY}

Optical spectra of the jet were obtained using the FOS on the Isaac Newton telescope at the Roque de los Muchachos Observatory, La Palma in January 1986.

From the [O III] and [N II] line ratios we calculate an electron density of $\sim 10^{6} \mathrm{~cm}^{-3}$ at a temperature of $\sim 8000 \mathrm{~K}$. These densities are above the critical density for the $\lambda 5008$ line. The [O III] 4363 to $\mathrm{H} \gamma$ ratio gave $10^{6} \mathrm{~cm}^{-3}$ at $8300 \mathrm{~K}$. Using the [S II] line ratios we obtain either $10^{6} \mathrm{~cm}^{-3}$ with $4600 \mathrm{~K}$ or $3.10^{5}$ $\mathrm{cm}^{-3}$ at $8000 \mathrm{~K}$. This might indicate that these lines are formed in regions with differing densities or temperatures. Our results compare well with those of Wallerstein and Greenstein (1980) (WG) who quote a density of $\sim 10^{6} \mathrm{~cm}^{-3}$ and $\mathrm{T}_{\mathrm{e}} \sim 10,000 \mathrm{~K}$ using forbidden line ratios. Using only the [O III] lines our density at a given temperature is somewhat lower than that of WG which might indicate expansion of the feature over the 9 year period between the observations. The results of Kafatos et al. (1986) based on UV line ratios give much lower densities at $\sim 10^{4} \mathrm{~cm}^{-3}$ at roughly the same temperature. This discrepancy is as yet unexplained. By obtaining emission measures from our optical data and comparing these with the emission measures of Kafatos et al. this question might be resolved. This work is now in progress. 


\section{POLARIMETRY}

It has been known for several years that $\mathrm{R} \mathrm{Aqr}$ is intrinsically polarized and that its polarization is variable. Here we present evidence for a correlation between the degree and angle of polarization and the Mira phase.

Firstly, Figure 1 shows the dramatic variation in the degree of polarization with the phase of observation. The variation is up to two orders of magnitude in the blue but very small in the red. All data runs show a dip in polarization across the $[\mathrm{O} \mathrm{III}]$ emission line at $\lambda 5008 \mathrm{~A}$. This might indicate that the central star is the source of polarization: when a large forbidden line contribution is present the relative polarization drops. The ratio of [O III] to nearby continuum is about 13; in the more heavily smoothed polarization data the depolarization is a factor of about 5 . This is at least qualitatively correct.

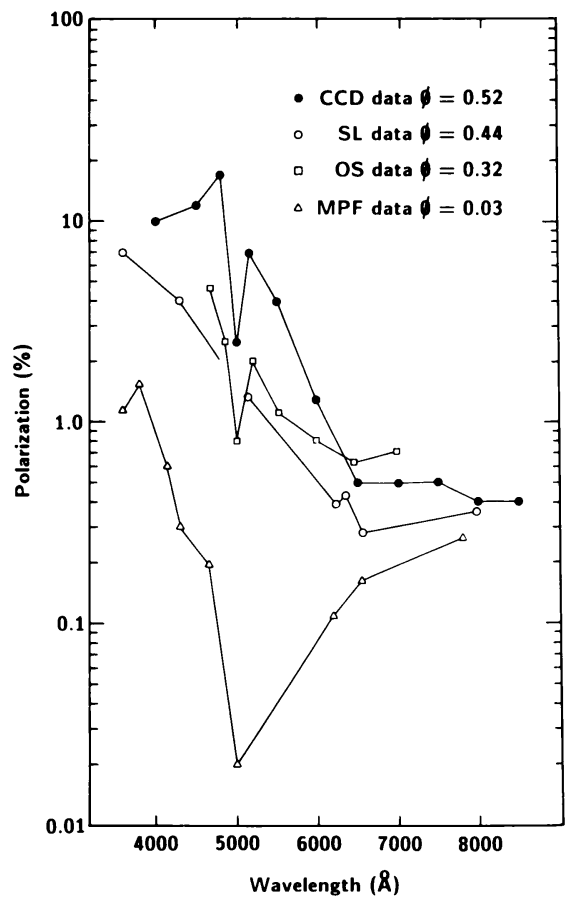

Figure 1. Degree of polarization against wavelength for four phases of R Aqr. CCD (Aspin et al., 1985), SL (Schulte-Ladbeck, 1985), OS (Oestreicher, unpublished) and MPF (Schwarz and Aspin, new data).



Figure 2. Degree (a) and angle (b) of U, V and $R$ polarization versus phase of $R$ Aqr.

Figure 2 shows the variation with phase of the degree (a) and angle (b) of polarization. The $U$ band measures are clearly correlated with the phase of the Mira, in the sense that maximum polarization coincides with minimum light. This behaviour is opposite to that of o Ceti (Shawl, 1974) where maximum polarization occurs near maximum light. The angle variation for $\mathrm{R}$ Aqr is also much larger than for o Cet but similar angular modulation is found for $\alpha$ Ori, a semi-regular M-type supergiant (Hayes, 1984; Schwarz, 1986).

Clearly, the modelling of phase-related polarization phenomena in Miras is not a straightforward task as also exemplified by the recently discovered, extremely complicated spectral features in the polarization of R Aqr (Aspin et al., 1985). 


\section{SUMMARY}

We have shown that the electron density and temperature of the jet in $R$ Aqr have not changed significantly since 1977 . A slight decrease in density is perhaps indicated. There is a significant difference between electron density as determined by optical and UV methods. This discrepancy is as yet unexplained. Collation of 17 years of polarization data shows Mira phase related behaviour of the $U$ band polarization and $\mathrm{U}, \mathrm{V}$ and $\mathrm{R}$ band angle changes. This is possibly because angle changes are generally position-dependent while degree of polarization changes tend to be strongly wavelength-dependent. The complex structure in polarization spectra cannot as yet be explained by any polarization models.

ACKNOWLEDGEMENTS. This work was supported by the SERC. HES would like to thank Prof. J. L. Culhane for his support and encouragement and Dr. J. R. Allington-Smith for donating some observing time. We acknowledge PATT for allocation of observing time at La Palma.

\section{REFERENCES}

Aspin, C., Schwarz, H.E., McLean, I.S., Boyle, R.P. (1985) Astron. Astrophys. 149, L21.

Baratta, G.B. et al. (1985) ESA SP239, pp95.

Hayes, D. P. (1984) Ap. J. Suppl. Ser. 55, 179.

Kafatos, M., Michalitsianos, A.G. (1982) Nature 298, 540.

Kafatos, M., Michalitsianos, A.G. Hollis, J.M. (1986), preprint.

Mauron, N. et al. (1985) Astron. Astrophys. 142, 413.

Schulte-Ladbeck, R. (1985) Astron. Astrophys. 142, 333.

Schwarz, H. E. (1986) Vistas in Astronomy, in press.

Serkowski, S. (1971) Kitt Peak Contr. No.554, 107.

Shawl, S. J. (1974) in : Planets, Stars and Nebulae studied with photopolarimetry, Arizona, UAP.

Stein, W.A. et al. (1969) Ap. J. 155, L3.

Wallerstein, G. (1986) Publ. Astron. Soc. Pac., 28, 118.

Wallerstein, G., Greenstein, J.L. (1980) Publ. Astron. Soc. Pac., 22, 275. 